\title{
Psychopathological Features in Alzheimer's Disease: Course and Relationship with Cognitive Status
}

\author{
Roee Holtzer, PhD, * Ming-Xin Tang, PhD, ${ }^{*+}$ D. P. Devanand, MD, $\neq$ Steven M. Albert, PhD, $*+$ \\ Domonick J. Wegesin, PhD, * Karen Marder, MD, MPH, ${ }^{*+}$ Karen Bell, MD, ${ }^{*+}$ Marilyn Albert, PhD, $\$$ \\ Jason Brandt, PhD, ll and Yaakov Stern, PhD, * $*$
}

OBJECTIVES: To examine the course, clinical correlates, and relationship between cognitive status and psychopathological features in patients with probable Alzheimer's disease $(\mathrm{AD})$ followed over a 5 -year period.

DESIGN: Cohort study with follow-up of 5 years.

SETTING: Patients were recruited at three sites: $91 \mathrm{pa}-$ tients at Columbia Medical Center, 84 at Johns Hopkins School of Medicine, and 61 at Massachusetts General Hospital.

PARTICIPANTS: Patients diagnosed with probable AD $(\mathrm{n}=236)$ enrolled in a longitudinal study (Predictors study).

MEASUREMENTS: Wandering/agitation, physical aggression, hallucinations, and delusions were evaluated at 6month intervals using the Columbia Scale for Psychopathology in Alzheimer's Disease. Descriptive analyses were used to provide estimates of prevalence and course of psychopathological features. General estimating equations determined the odds of having any of the four psychopathological behaviors as a function of cognitive status. Markov analyses provided 6-month transition probabilities for psychopathological behaviors given patients' cognitive status and the presence or absence of such behaviors in the previous evaluation.

RESULTS: For wandering/agitation, prevalence (39-57\%) and persistence increased as a function of time and decrement in cognitive status. Physical aggression was less prevalent $(6-22 \%)$ and increased as a function of cognitive decline but tended to persist only in the more severely impaired patients. Delusions (34-49\%) reached a peak at

From the *Sergievsky Center and Departments of ${ }^{\dagger}$ Neurology and ${ }^{\ddagger}$ Psychiatry, Columbia University College of Physicians and Surgeons, New York, New York; 'Departments of Psychiatry and Neurology, Massachusetts General Hospital, Harvard Medical Center, Boston, Massachusetts; and IDepartment of Psychiatry and Behavioral Sciences, Johns Hopkins University, Baltimore, Maryland.

This work was supported in part by National Institutes of Health and National Institute of Aging Grants AG07370, RR00645, and MH55735

Address correspondence to Yaakov Stern, PhD, Sergieversusky Center, 19th floor, 630 West 168th Street, P \& S Box 16, New York, NY 10032. E-mail: ys11@columbia.edu the second year and then declined. The odds of delusions were maximal with intermediate decline but remained persistent regardless of cognitive status. Hallucinations, despite some fluctuations, were relatively stable during the follow-up period $(8-17 \%)$ and moderately persistent.

CONCLUSION: Psychopathological features, particularly wandering/agitation and delusions, in $\mathrm{AD}$ were common throughout the disease course. The natural history and persistence of the four psychopathological features varied. These findings provide important information to clinicians and caregivers regarding the course, predictability, and possible treatment of psychopathological behaviors in patients with probable AD. J Am Geriatr Soc 51:953-960, 2003.

Key words: psychopathological features; cognition; Alzheimer's disease

$\mathrm{P}$ sychopathological features such as delusions and hallucinations are integral elements of Alzheimer's disease (AD) and present significant problems for family members and caregivers of demented patients. ${ }^{1}$ Estimates of the prevalence of delusions in $\mathrm{AD}$ have ranged from $10 \%$ to $73 \%,{ }^{2}$ with an average of approximately $35 \% .^{3}$ Prevalence rates of hallucinations have ranged from $3 \%$ to $49 \% .^{3}$ These estimates vary because of different methods by which delusions and hallucinations were diagnosed and because of differences in study populations. ${ }^{4}$ Also, crosssectional studies ${ }^{5-7}$ are not sensitive to changes in the prevalence of psychotic symptoms as a function of disease progression. There have been few longitudinal studies concerning the course and prevalence of psychopathological behaviors in $\mathrm{AD}$. The authors previously reported on the course of psychopathological features including delusions and hallucinations in a prospective sample of patients with probable AD followed for a 3-year period. ${ }^{8}$

Evidence for the relationship between psychotic symptoms, the severity of cognitive deficits, and the rate of cognitive decline in $\mathrm{AD}$ has been inconsistent. Several studies have demonstrated that the presence of psychotic symp- 
toms is related to greater severity of cognitive deficits ${ }^{9-16}$ and to accelerated cognitive decline, ${ }^{9-11,14,16,17}$ but other studies have failed to show this relationship ${ }^{18-20}$ or have presented equivocal findings. ${ }^{21-23}$ Furthermore, studies examining delusions and hallucinations separately appear to be inconsistent as to whether accelerated cognitive decline is related to hallucinations only ${ }^{9}$ or to delusions only. ${ }^{12}$

Wandering occurs in up to $65 \%$ of patients in nursing homes or dementia-based clinic samples ${ }^{24}$ and in approximately $35 \%$ of community samples. ${ }^{15}$ Agitation has been reported in up to $50 \%$ of $\mathrm{AD}$ patients in outpatient clinics ${ }^{25}$ and nursing homes. ${ }^{26,27} \mathrm{~A}$ recent prospective study ${ }^{8}$ showed that the prevalence of wandering or agitation ranged from $38 \%$ to $62 \%$ during a 3 -year follow-up. Also, wandering and agitation appear to be related both to the severity of cognitive deficits $8,24,28$ and to cognitive decline. ${ }^{19}$

Estimates of the prevalence of physical aggression in $\mathrm{AD}$ range from $15 \%$ to $20 \%{ }^{29}$ but reach $30 \%$ in a clinical sample of moderately to severely demented patients. ${ }^{30} \mathrm{~A}$ recent prospective study of patients with probable $\mathrm{AD}^{8}$ showed that the prevalence of physical aggression ranged from $6.4 \%$ to $20.5 \%$ over a 3 -year period. Although physical aggression is related to other psychopathological disturbances, ${ }^{30-34}$ evidence for its relationship to cognitive status has been examined in only a few studies, yielding mixed results. Physical aggression was related to greater severity of cognitive deficits in some studies ${ }^{8,29}$ but not in others. ${ }^{32-34}$

The present study examined the course, clinical correlates, and relationship to decrements in cognitive status of psychopathological behaviors in $\mathrm{AD}$ using the same prospective sample of patients previously reported in a 3-year follow-up. ${ }^{8}$ Specifically, the goal of this investigation was threefold: first, to evaluate the prevalence and course of delusions, hallucinations, wandering/agitation, and physical aggression over a 5 -year period in patients with probable AD; second, to determine the odds of presenting with behavioral disturbances as a function of cognitive status; and third, to examine whether psychopathological disturbances persist over time and whether the degree of persistence is related to cognitive status. To the authors' knowledge, this is the first study that provides such prospective data for $\mathrm{AD}$ patients followed from mild to severe stages of the disease.

Information regarding the course and predictability of psychopathological behaviors in $\mathrm{AD}$ is of significant value and importance to clinicians and caregivers of demented patients. Furthermore, pharmacological treatment of psychopathological features in AD has been shown to be effective. ${ }^{35-38}$ Hence, knowledge regarding the course, persistence, and relationship to disease stage of specific psychopathological behaviors can improve the efficacy of such treatment. ${ }^{39}$

\section{METHODS}

\section{Participants}

Two hundred thirty-six patients, enrolled in the Predictors study, participated in the present investigation. All patients were diagnosed with probable AD. ${ }^{40,41}$ At entry into the study, patients were required to have relatively mild dementia, which was operationalized as modified MiniMental State Examination (mMMSE) score of 30 (equiva- lent to 16 on the Folstein MMSE). Patients were recruited at three sites: 91 patients at Columbia Medical Center, 84 at Johns Hopkins School of Medicine, and 61 at Massachusetts General Hospital. At the Columbia site, patients were recruited from the Memory Disorder Center and from doctors' private offices through the Alzheimer's Disease Research Center. Each consecutive patient who met the inclusion/exclusion criteria of the study was included, with the exception of those who did not consent to participate or who lived too far away and were unable to return to the hospital for regular follow-up. At the Johns Hopkins site, patients were recruited from the Dementia Research Center and from private physicians. For the Massachusetts General Hospital site, patients were recruited from the Geriatric Neurobehavioral Center, an outpatient service. In addition, 14 patients were recruited from a longterm care facility, and eight patients also entered a physostigmine study.

The inclusion/exclusion criteria and evaluation procedures of the Predictors study have been fully described elsewhere. ${ }^{42,43}$ With respect to psychiatric history, exclusion criteria included a history of schizophrenia or schizoaffective disorder, electroconvulsive therapy within the preceding 2 years or a history of 10 or more electroconvulsive treatments in any one course, alcohol or drug dependence during the preceding 5 years, primary affective disorder within 1 year before onset of dementia, and evidence of other causes of dementia.

In the Predictors study, patients are evaluated every 6 months. The total number of patients followed from the initial evaluation through the fifth year was 236, 205, $170,154,125$, and 102 , respectively. Of the 134 patients lost during the 5-year follow-up, 118 died. Reasons for loss of contract with the remaining 16 patients $(6.7 \%$ of the entire sample) included refusal to participate, relocation, and medical condition that did not allow for continued follow-up. The mean number \pm standard deviation of 6 -month visits for the entire sample was $6.7 \pm 5.5$, indicating that, on average, patients have been followed for approximately 3.5 years.

Patients' mental status, dependence, and daily functional competence were evaluated as a part of a neurological examination using the mMMSE, ${ }^{44}$ Dependence Scale, ${ }^{45}$ and Blessed Functional Activities Scale (BFAS). ${ }^{46}$

\section{Measures}

\section{Psychopathology}

Psychopathological behaviors were assessed using the Columbia University Scale for Psychopathology in Alzheimer's Disease (CUSPAD). ${ }^{47}$ The CUSPAD is a short semistructured rating scale that a clinician or research technician can administer to an informant. The frame of inquiry is the month before examination. Most items are scored dichotomously (i.e., symptom is present or absent). Psychometric properties of this instrument have been reported. ${ }^{47}$ Interrater reliability for the major symptom categories was established for concurrent rating of a single interview $(\kappa=$ $0.74-1.00)$ and for separate interviews $(\kappa=0.53-0.73)$. Correlations between major symptom categories were low and statistically insignificant, indicating good divergent validity. The CUSPAD was developed primarily to exam- 
ine phenomenological features, and most items lack quantitative rating of severity. Consequently, partial response to treatment and fluctuation over time in item severity are not detected with this instrument.

A trained research technician administered the CUSPAD to an informant at the initial evaluation and at subsequent 6-month intervals. Four dichotomous CUSPAD categories-delusion (paranoid, misidentification, somatic, abandonment), hallucination (visual, auditory, tactile, olfactory), wandering/agitation (i.e., restlessness or increased motor activities), and physical aggression (including threatening behavior)-were used for statistical analyses. The presence of any symptom within a category led to a positive score for that category. As previously reported, ${ }^{8}$ a broad definition of a delusion (i.e., the patient does not accept the caregiver's correction for the false belief) and the presence of a vague or clear hallucination were used to determine category status (i.e., present or absent).

\section{Cognition}

Cognition was assessed using the modified version of the MMSE. ${ }^{48}$ Modifications to the original MMSE include the addition of digit span forward and backward, ${ }^{49}$ two additional calculation items, recall of the current and four previous presidents of the United States, confrontation naming of 10 items from the Boston Naming Test, ${ }^{50}$ one additional sentence to repeat, and one additional figure to copy. The mMMSE has a maximum of 57 points, with lower scores indicating poorer cognitive function. The psychometric properties for the mMMSE have been well described. ${ }^{44}$

\section{Statistical Analyses}

\section{Descriptive Statistics}

The percentage of patients per year experiencing delusions, hallucinations, wandering/agitation, and physical aggression was reported for a 5-year period and delineated in relation to mean mMMSE scores. The prevalence rates, based on all available patient visits, were compared with those obtained for a subset of 48 patients who had completed all 6-month-interval evaluations during the 5-year follow-up. This was done to assure that changes in yearly prevalence rates for the entire sample did not reflect attrition, missed appointments, and death.

The specific nature of the relation between cognitive function and the prevalence of psychopathological behaviors was addressed in the general estimating equation (GEE) analyses described below.

\section{General Estimating Equations}

GEEs were used to calculate the odds of having each of the psychopathological behaviors as a function of cognitive status using the entire sample $(\mathrm{n}=236)$ with all available patient visits. This statistical method takes into account the multiple visits per patient as well as the likelihood that an individual's characteristics correlate with each other over time. The repeated measures per patients (up to 236 month visits) are treated as a cluster. A second advantage of GEE is that it takes into account the status or changing value of each covariate at each visit. In the current GEE model, five mMMSE percentile groups $(0-20 \%$ ile, 21-
$40 \%$ ile, $41-60 \%$ ile, $61-80 \%$ ile, $81-100 \%$ ile) served as the categorical independent variable. Patients' mMMSE group membership was determined for each available 6month visit based on their mMMSE scores. The top percentile mMMSE group served as a reference group against which the four other mMMSE percentile groups were compared. The presence or absence of delusions, hallucinations, wandering/agitation, and physical aggression served as the dichotomous dependent variable. Analyses controlled for age, education, and sex.

Additional GEE models for delusions and hallucinations controlled for the effect of psychotropic medications on psychotic symptoms and cognition.

\section{Markov Analyses}

Markov analyses were used to evaluate 6-month-interval transition probabilities of psychopathological behaviors during the 5 -year follow-up. Only the 48 patients who had completed all 6-month visits during the 5-year follow-up were included. Patients were assigned at each 6-month evaluation to one of five mMMSE percentile groups (0$20 \%$ ile, $21-40 \%$ ile, $41-60 \%$ ile, $61-80 \%$ ile, $81-100 \%$ ile). In the current model, mMMSE status and the presence or absence of psychopathological behaviors at the preceding 6-month visit served as independent variables used to determine the transition probabilities to the specified outcome (i.e., maintaining or developing psychopathological behaviors) at the next visit.

\section{RESULTS}

Of the 236 participants, $59 \%$ were female; the majority of patients were white. Mean \pm standard deviation age and education, in years, were $72.7 \pm 9.2$ and $13.0 \pm 3.6$, respectively. Mean baseline mMMSE was $37.2 \pm 6.1$, indicating an overall mild level of cognitive impairment. The modal dependence level at baseline was $2(54 \%$ of the patients), suggesting that, initially, most patients required limited home care or supervision. ${ }^{45}$ Mean total BFAS total score at baseline was $8.0 \pm 3.4$, indicating mild level of functional impairments. ${ }^{51}$

\section{Descriptive Statistics}

The percentage of patients per year experiencing wandering/ agitation, physical aggression, hallucinations, and delusions during the 5-year follow-up is presented in Figure 1. These findings are presented in relation to yearly mean mMMSE scores.

Inspection of Figure 1 reveals that the symptoms of wandering/agitation and physical aggression tended to increase as a function of time and disease progression. The prevalence of hallucinations tended to increase slightly over the 5-year period. For delusions, maximal prevalence appeared to occur in the second year, with a subsequent decline. Only seven patients were determined to be mute at the end of the 5-year follow-up, suggesting that the decline in the prevalence of delusions (after a peak) was not solely due to patients' inability to communicate delusional experiences.

The percentage of patients experiencing psychopathological behaviors also was calculated using the subset of 48 patients who had completed all 6-month visits during the 5-year follow-up. This was initiated to assure that changes in yearly prevalence rates did not reflect fluctua- 

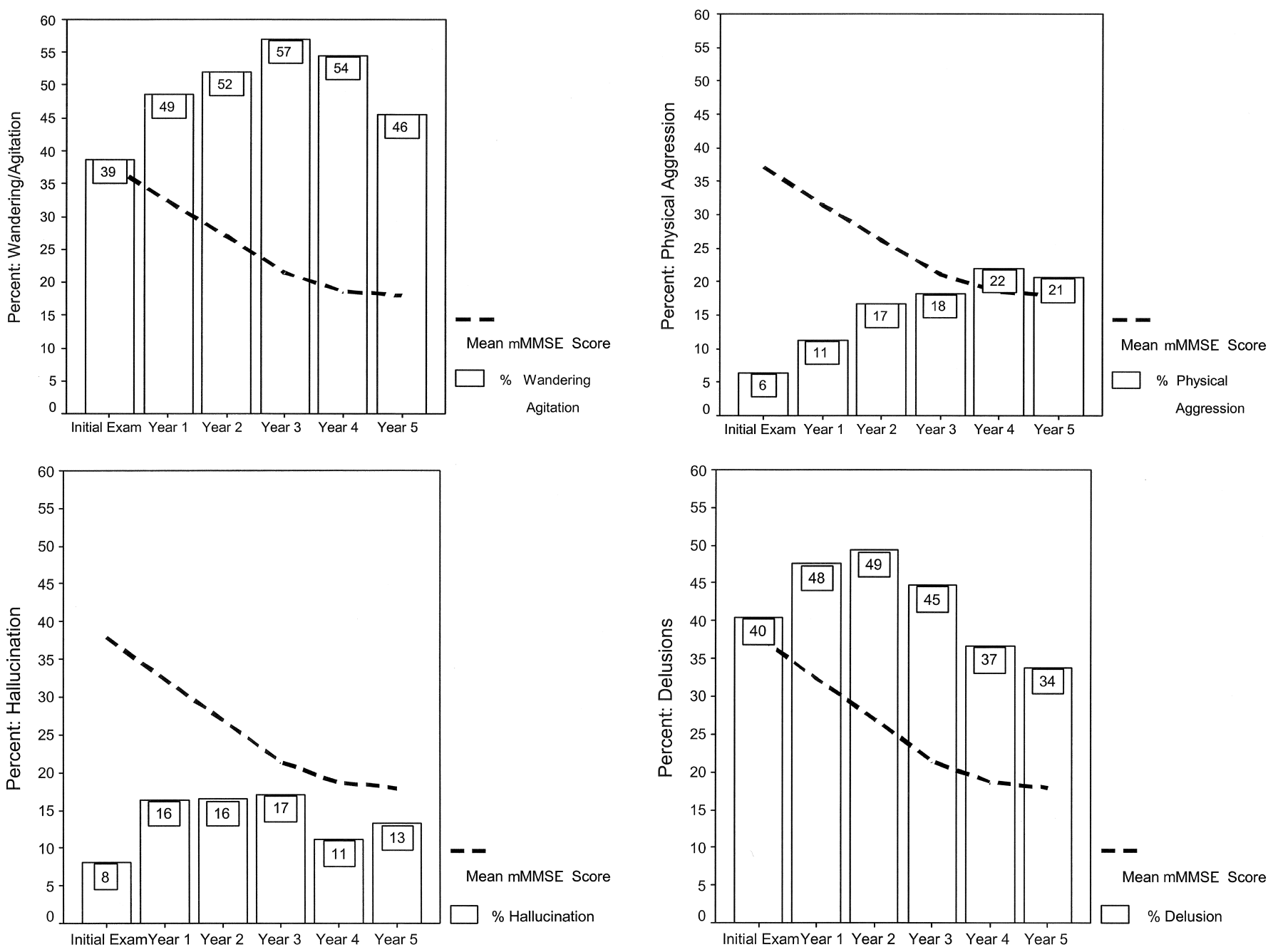

Figure 1. Percentage of patients deemed to present with (A) wandering/agitation, (B) physical aggression, (C) hallucination, and (D) delusions during the initial evaluation and 5-year follow-up. mMMSE = Modified Mini-Mental State Examination.

tions in the number of patients evaluated each year. Indeed, the two analyses were similar in terms of the prevalence and changes over time in the prevalence rates of the four psychopathological behaviors examined.

It appears that decline in mMMSE scores was associated with an increase in the percentage of patients experiencing any one of the four psychopathological behaviors examined, but in these descriptive analyses, mMMSE data were delineated as a sample mean per year, limiting inferences regarding the association between the severity of mMMSE decline and changes in the prevalence of psychopathological behaviors. Therefore, the odds of presenting with psychopathological symptoms, as a function of cognitive status, were examined in the GEE analyses described below.

\section{GEE Analyses}

The mMMSE group status, for all available 6-month visits, served as the categorical independent variable. The highest percentile group (81-100\%ile) constituted a reference group against which the four lower mMMSE percentile groups were compared. The presence or absence of each psychopathological category for each 6-month visit served as the dichotomous dependent variable. Table 1 presents significance level and odds ratio (OR) (i.e., associated risk) of exhibiting psychopathological behaviors as a function of cognitive status.

Inspection of Table 1 revealed that the odds of exhibiting wandering/agitation and physical aggression increased as a function of decrements in cognitive status. Membership in the 61 to 80 percentile, 41 to 60 percentile, 21 to 40 percentile, and 0 to 20 percentile mMMSE groups was associated with a $1.5,2.0,3.3$, and 4.2-fold increase, respectively, in the odds of having wandering/agitation, relative to the reference group. For physical aggression, membership in the 61 to 80 percentile, 41 to 60 percentile, 21 to 40 percentile, and 0 to 20 percentile mMMSE groups was associated with a 3.5-, 3.1-, 3.6-, and 9.0-fold increase, respectively, in the odds of having such behavior. Lower mMMSE percentile group status also was associated with higher odds (range of 2- to 3.3-fold relative to the reference group) of having hallucinations. The odds of having delusions increased by 1.4-, 2.3-, and 2.4-fold in the 61 to 80 percentile, 41 to 60 percentile, and 21 to 40 percentile groups, respectively, compared with the refer- 
Table 1. Comparison of Four Modified Mini-Mental State Examination (mMMSE) Percentile Groups with Reference Group (81-100\%ile mMMSE group)

\begin{tabular}{|c|c|c|c|c|c|c|c|c|c|}
\hline \multirow{3}{*}{$\frac{\text { Percentile }}{81-100}$} & \multirow{3}{*}{$\begin{array}{c}\text { mMMSE } \\
\begin{array}{c}\text { Corresponding } \\
\text { Range of Scores }\end{array} \\
39-57\end{array}$} & \multicolumn{2}{|c|}{$\begin{array}{l}\text { Wandering/ } \\
\text { Agitation }\end{array}$} & \multicolumn{2}{|c|}{$\begin{array}{l}\text { Physical } \\
\text { Aggression }\end{array}$} & \multicolumn{2}{|c|}{ Hallucination } & \multicolumn{2}{|c|}{ Delusion } \\
\hline & & \multicolumn{8}{|c|}{ Odds Ratio $P$-value } \\
\hline & & 1 & Reference & 1 & Reference & 1 & Reference & 1 & Reference \\
\hline $61-80$ & 33-38 & 1.5 & .0568 & 3.5 & .0016 & 2.0 & .0287 & 1.4 & .0314 \\
\hline $41-60$ & $26-32$ & 2.0 & .0064 & 3.1 & .0001 & 2.6 & .0009 & 2.3 & $<.0001$ \\
\hline
\end{tabular}

ence group. However, the odds of having delusions in the 0 to 20 percentile of the mMMSE group were not significantly different from the odds reported for the reference group. The results of the GEE analyses appeared consistent with the descriptive data described earlier regarding the course of each of the four psychopathological categories and its relationship with cognitive function.

The effect of psychotropic medications on psychotic symptoms and cognition was addressed as well. GEE analyses for delusions and hallucination were repeated under two different exclusionary conditions. First, only visits during which patients were on psychotropic medications were excluded. Second, patients were excluded if psychotropic medications were used at any point during the course of their follow-up. Compared with the initial models, the association between cognitive status and delusions and hallucinations remained similar.

\section{Markov Analysis}

Markov analysis was used to determine 6-month transitional probabilities of psychopathological behaviors. Table 2 presents transition probabilities for each psychopathological behavior as a function of mMMSE status and whether the behavior was present in the preceding visit.

Transition probabilities range from 0 to 1 . For example, transition probability of 0.20 for "new onset" of wandering/agitation in the 81 to 100 percentile mMMSE group, indicates that $20 \%$ of patients not reported to ex- hibit wandering/agitation in the preceding 6-month visit (n - 1), manifested such behavior at the next 6-month visit (n). A transition probability of 0.54 for "persistent" status of wandering/agitation in the 81 to 100 percentile mMMSE group indicates that $54 \%$ of the patients reported to exhibit wandering/agitation in the preceding 6month visit $(\mathrm{n}-1)$ manifested such behavior at the next 6-month visit $(\mathrm{n})$.

Table 2 reveals that mMMSE status and the presence of psychopathological behaviors at one 6-month visit were significant predictors of transitional probabilities at the next 6-month visit.

For wandering/agitation, transition probabilities increased from 0.20 in the 81 to 100 percentile mMMSE group to 0.48 in the 0 to 20 percentile mMMSE group when this behavior was not present in the preceding visit. The transition probabilities for these high and low mMMSE percentile groups rose to 0.54 and 0.80 , respectively, when this behavior was present in the preceding visit. Transitional probabilities for physical aggression also increased as a function of decrements in cognitive status $(0.05$ and 0.26 in the $81-100 \%$ ile and $0-20 \%$ ile mMMSE groups, respectively) in the absence of this behavior at the preceding 6-month visit. When physical aggression was present in the preceding interval, the transition probabilities rose to 0.27 and 0.69 in the high and low mMMSE percentile groups, respectively. Furthermore, it is noteworthy that physical aggression appeared to be persistent only

Table 2. Markov Analysis-Transition Probabilities for Wandering/Agitation, Physical Aggression, Hallucination, and Delusion Given Modified Mini-Mental State Examination (mMMSE) Group Status and Whether Behavior was Present in Preceding 6-Month Visit

\begin{tabular}{|c|c|c|c|c|c|c|c|c|c|}
\hline \multirow[b]{2}{*}{ Percentile } & \multirow{2}{*}{$\begin{array}{c}\text { mMMSE } \\
\text { Corresponding } \\
\text { Range of Scores }\end{array}$} & \multicolumn{2}{|c|}{ Wandering/Agitation } & \multicolumn{2}{|c|}{ Physical Aggression } & \multicolumn{2}{|c|}{ Hallucination } & \multicolumn{2}{|c|}{ Delusion } \\
\hline & & New Onset & Persistent & New Onset & Persistent & New Onset & Persistent & New Onset & Persistent \\
\hline $81-100$ & $39-57$ & 0.20 & 0.54 & 0.05 & 0.27 & 0.03 & 0.14 & 0.18 & 0.76 \\
\hline $61-80$ & $33-38$ & 0.24 & 0.59 & 0.04 & 0.20 & 0.12 & 0.42 & 0.17 & 0.75 \\
\hline $41-60$ & $26-32$ & 0.35 & 0.72 & 0.04 & 0.22 & 0.07 & 0.30 & 0.20 & 0.78 \\
\hline $21-40$ & $14-25$ & 0.49 & 0.81 & 0.18 & 0.59 & 0.15 & 0.50 & 0.23 & 0.82 \\
\hline $0-20$ & $0-13$ & 0.48 & 0.80 & 0.26 & 0.69 & 0.12 & 0.43 & 0.10 & 0.64 \\
\hline
\end{tabular}

New onset $=$ not present in previous visit; Persistent $=$ present in previous visit. 
in the two lower mMMSE percentile groups. Six-month transitional probabilities for hallucinations, although tending to increase as a function of lower mMMSE percentile group status, appeared to fluctuate when hallucinations were present or absent in the preceding 6-month interval. Membership in the 81 to 100 percentile mMMSE group was associated with the lowest transition probabilities for the new onset and persistent conditions. Six-month transition probabilities for delusions increased as a function of lower mMMSE percentile group status in the new onset and persistent conditions but then decreased in the 0 to 20 percentile mMMSE group. Additionally, delusions were persistent in all mMMSE percentile groups.

\section{DISCUSSION}

The present study examined the course and prevalence of wandering/agitation, physical aggression, delusions, and hallucinations in patients with probable AD over a 5-year period. The odds of presenting these psychopathological behaviors were examined in relation to cognitive status. Also, 6-month transition probabilities for each psychopathological behavior were determined. The current findings replicate and extend previously published data for the same prospective sample of patients. ${ }^{8}$

The results are consistent with previous research ${ }^{2-7,15,24-27}$ demonstrating that behavioral disturbances are common in AD. Specifically, wandering/agitation increased from a baseline prevalence rate of $39 \%$ to a peak of $57 \%$. Physical aggression, although not as prevalent, also increased as a function of the disease course and peaked at $22 \%$. The prevalence of hallucinations remained relatively stable during the 5 -year follow-up, ranging from $8 \%$ to $17 \%$. Delusions increased during the first and second years, reaching a peak of $49 \%$, with a subsequent drop to $34 \%$ at the end of the 5-year follow-up. These results may explain some of the reported variability of psychopathological behaviors in $A D$, because the range of prevalence rates for these four behavioral disturbances varied substantially as a function of time and disease course. Despite the noteworthy increase in the prevalence of wandering/agitation, physical aggression, hallucinations, and delusions as a function of the disease course, these behavioral disturbances were not time- or disease-phase limited. Consistent with previous research, ${ }^{52}$ they appeared throughout the 5 -year follow-up. It should be emphasized that changes in prevalence of the different psychopathological features over the course of follow-up are not likely to reflect fluctuations in the number of patients evaluated each year. Indeed, such prevalence rates were similar in a subset of patients who were present at all 6-month evaluations.

The findings of this investigation provided strong support to previous research in favor of the association between cognitive dysfunction and the presence of psychotic symptoms ${ }^{9-17}$ wandering/agitation $8,19,24,28$ and physical aggression. ${ }^{8,29}$ GEE analyses revealed that the odds of experiencing these psychopathological disturbances increased significantly as a function of declining cognitive status. A conservative approach excluding all patients prescribed psychotropic medications did not attenuate the relation between psychotic symptoms and cognitive status. Furthermore, with the exception of four participants, patients who were prescribed cholinesterase inhibitors at some point during the follow-up also were prescribed psychotropic medications and consequently were excluded from these analyses as well.

In the present study, the mMMSE served as the sole measure of cognition, limiting the extent and specificity of the conclusions regarding the association between cognitive function and psychopathological features in $\mathrm{AD}$. Of interest is whether decline in specific cognitive domains can provide additional information beyond that available from the mMMSE, with respect to the risk of experiencing psychopathological symptoms during the disease course. A recent study ${ }^{10}$ revealed that decline in measures of attention, construction, and fluency, which are considered sensitive to frontal lobe dysfunction, were related to an increase in the risk of developing psychosis in patients with probable AD. These results, in concert with the strong association between cognitive dysfunction and psychopathological features documented in the presented study, may be viewed as supportive evidence of the hypofrontality model of psychosis in AD. This model argues that patients with probable $\mathrm{AD}$ who experience a disproportionate dysfunction of frontal circuitry are at greater risk of developing psychosis. Physical aggression and wandering/agitation may be considered symptoms of compromised ability to inhibit responses, a cognitive behavioral deficit that has been attributed to frontal dysfunction as well. Taken together, the evolution and progression of psychopathological features in $\mathrm{AD}$ may be related, at least to some extent, to damage in frontal regions.

The present study was consistent with previous research ${ }^{8}$ revealing that psychopathological behaviors were not only prevalent in $\mathrm{AD}$ but also persistent. That is, patients who presented with behavior disturbances at one 6-month evaluation were more likely to exhibit the same disturbance at the following 6-month evaluation relative to those patients who did not present with the same behavioral disturbance at the previous evaluation. Nevertheless, the degree of persistence of symptoms differed among the four psychopathological behaviors and also varied as a function of cognitive status. The transition probabilities for wandering/ agitation revealed that this behavior was persistent in all mMMSE percentile groups but that the level of persistence increased as cognitive status decreased. Physical aggression appeared to be persistent only in the two lower mMMSE percentile groups. Hallucinations were not persistent in the 81 to 100 percentile mMMSE group but appeared moderately persistent (transition probabilities ranged from 0.30 to 0.50 ) in the other mMMSE percentile groups. Delusions were persistent irrespective of cognitive status, although a reduction in transition probabilities was noted in the 0 to 20 percentile mMMSE group.

Data concerning the persistence of psychopathological features have some limitations in that Markov analyses did not control for psychotropic medications, because of the small number of patients $(n=48)$. Furthermore, the CUSPAD was developed primarily to examine phenomenological features, and most items lack quantitative rating of severity. Hence, this instrument would have missed reduction in symptoms severity due to pharmacological intervention without complete remission.

There were some noteworthy differences among the four psychopathological behaviors in terms of their preva- 
lence and relation to cognitive status. Wandering/agitation and physical aggression, despite differences in prevalence rates, tended to increase as a function of disease course and decrements in cognitive function. The dramatic increase in the odds of exhibiting physical aggression in the 0 to 20 percentile mMMSE group relative to the reference group (9.0-fold) and the persistence of this behavior (transitional probabilities $=0.69$ ) in this group is noteworthy. It appears that patients with severe cognitive dysfunction who were physically aggressive in the past are especially prone to exhibit this behavior again. Hallucinations, despite some fluctuations, remained relatively stable across the duration of the follow-up. Nonetheless, the odds of having hallucinations increased as cognitive status decreased.

The prevalence of delusions peaked at the end of the second year, followed by a subsequent drop. An examination of descriptive and GEE analyses suggests that the relationship between the prevalence of delusions and cognitive status is not linear. The odds of having delusions increased as a function of cognitive dysfunction but then dropped when cognitive status reached a bottom level. The reason for this phenomenon is not clear. As indicated by these data, inability to communicate delusional experiences does not appear to provide a satisfactory explanation. It can be speculated that delusions require a relatively complex cognitive substrate not available when cognitive function is most severely compromised. Nonetheless, the prevalence of delusions remained at $34 \%$ at the end of the 5 -year period. Future research may provide insight into whether the content of delusions is simplified as the disease progresses. Hence, the reduction in the prevalence of delusions over time may be explained if complex delusions are not experienced at the end stages of AD.

The longitudinal design of this study has significant advantages over cross-sectional studies when evaluating the manifestation and progression of psychopathological features over time and in relation to cognitive status in AD. Specifically, when assessed longitudinally, the increase in symptoms can be attributed directly to disease progress and the severity of cognitive dysfunction. In contrast, findings from cross-sectional studies cannot be used to make inferences regarding such disease processes. (For a recent review concerning the limitations of cross-sectional studies with respect to disease processes see Kraemer et al. ${ }^{53}$ ) Furthermore, the findings of the present study afford valuable information to clinicians and caregivers of demented patients regarding the course and predictability of psychopathological behaviors in AD. For example, the high frequency and persistent nature of wandering/agitation and delusions indirectly suggests that treatment for these symptoms may need to be prolonged. Also, physical aggression, relatively infrequent early in the disease, appears to persist only in late phases. This suggests that once treatment for this behavior begins it may need to be administered continuously. Finally, the findings of this study were based on a relatively large prospective sample, allowing for conclusions that are likely applicable to many $\mathrm{AD}$ patients. Nevertheless, the Caucasian predominance and relatively high education level in this sample may limit the generalizability of the findings to patients of different ethnic origins with less education.

In summary, this prospective study demonstrated that psychopathological behaviors, particularly wandering/ agitation and delusions, in AD were common. The odds of presenting with any one of the four psychopathological behaviors increased significantly as a function of decline in cognitive status. Finally, transition probabilities showed that psychopathological behaviors were persistent but that the degree of persistence varied as a function of cognitive status and type of behavior.

\section{REFERENCES}

1. Finkel SI, Silva JC, Coben GD et al. Behavioral and psychological symptoms of dementia. Am J Geriatr Psychiatry 1998;6:97-100.

2. Leuchter AF, Spar JE. The late-onset psychosis. J Nerv Mental Dis 1985;173: 488-494.

3. Wragg R, Jeste D. Overview of depression and psychosis in Alzheimer's disease. Am J Psychiatry 1988;146:577-587.

4. Rao V, Lyketsos CG. Delusions in Alzheimer's disease: A review. J Neuropsychiatry Clin Neurosci 1998;10:373-382.

5. Bassiony MM, Steinberg MS, Warren A et al. Delusions and hallucinations in Alzheimer's disease: Prevalence and clinical correlates. Int J Geriatr Psychiatry 2000;15:99-107.

6. Ballard CG, Saad KP, Atel A et al. The prevalence and phenomenology of psychotic symptoms in dementia sufferers. Int J Geriatr Psychiatry 1995;10: 477-485.

7. Rockwell E, Jackson E, Gary V et al. A study of delusions in a large cohort of Alzheimer's disease patient. Am J Geriatr Psychiatry 1994;2:157-164.

8. Devanand DP, Jacobs DM, Tang M-X et al. The course of psychopathological features in mild to moderate Alzheimer disease. Arch Gen Psychiatry 1997;54:257-263.

9. Wilson RS, Gilley DW, Bennet DA et al. Hallucinations, delusions, and cognitive decline in Alzheimer's disease. J Neurol Neurosurg Psychiatry 2000; 69:172-177.

10. Paulsen JS, Salmon DP, Thal LJ et al. Incidence of and risk factors for hallucinations and delusions in patients with probable AD. Neurology 2000;54: 1965-1971.

11. Frisoni GB, Rozzini L, Gozzetti A et al. Behavioral syndromes in Alzheimer's disease. Description Correlates. Dement Geriatr Cogn Disord 1999;10:130 138.

12. Haput M, Romero B, Kurz A. Delusions and hallucinations in Alzheimer's disease: Results from a two-year longitudinal study. Int J Geriatr Psychiatry 1996;11:965-972.

13. Levy ML, Cummings JL, Fairbanks LA et al. Longitudinal assessment of symptoms of depression, agitation, and psychosis in 181 patients with Alzheimer's disease. Am J Psychiatry 1996;153:1438-1443.

14. Stern Y, Albert M, Brandt J et al. Utility of extrapyramidal signs and psychosis as predictors of cognitive and functional decline, nursing home admission, and death in Alzheimer's disease. Neurology 1994;44:2300-2307.

15. Devanand DP, Brockington CD, Moody BJ et al. Behavioral syndromes in Alzheimer's disease. Int Psychogeriatr 1992;4 (Suppl. 2):161-184.

16. Stern Y, Mayeux R, Sano M et al. Predictors of disease course in patients with probable Alzheimer's disease. Neurology 1987;37:1649-1653.

17. Cooper JK, Mungas D, Weiler P. Relation of cognitive status and abnormal behaviors in Alzheimer's disease. J Am Geriatr Soc 1990;38:867-870.

18. Kotrla KJ, Chacko RC, Harper RG et al. Clinical variables associated with psychosis in Alzheimer's disease. Am J Psychiatry 1995;152:1377-1379.

19. Miller T, Tinkelberg J, Brooks J et al. Selected psychiatric symptoms associated with rate of cognitive decline in patients with Alzheimer's disease. J Geriatr Psychiatry Neurol 1993;6:235-238.

20. Burns A, Jacoby R, Levy R. Psychiatric phenomena in Alzheimer's disease. Br J Psychiatry 1990;157:72-76.

21. Bylsma FW, Folstein MF, Devanand DP et al. Delusions and patterns of cognitive impairments in Alzheimer's disease. Neuropsychiatry Neuropsychol Behav Neurol 1994;7:98-103.

22. Becker D, Hershkowitz M, Maidler N et al. Psychopathology and cognitive decline in dementia. J Nerv Ment Dis 1994;182:701-703.

23. Mortimer JA, Ebbitt B, June SP et al. Predictors of cognitive and functional decline in patients with probable Alzheimer's disease. Neurology 1992;42: 1689-1696.

24. Logsdon RG, Terri L, McCurry SM et al. Wandering: A significant problem among community-residing individuals with Alzheimer's disease. J Gerontol B Psychol Sci Soc Sci 1998;53B:P294-P299.

25. Teri L, Larson EB, Reifler BV. Behavioral disturbances in dementia of the Alzheimer's type. J Am Geriatr Soc 1988;36:1-6.

26. Chandler JD, Chandler JE. The prevalence of neuropsychiatric disorders in a nursing home population. J Geriatr Psychiatry Neurol 1988;1:71-76. 
27. Cohen-Mansfield J, Marx MS, Rosental AS. A description of agitation in a nusing home. Gerontology 1988;3:M77-M84.

28. Cooper JK, Mungas D. Risk factor and behavioral differences between vascular and Alzheimer's dementias: The pathway to end-stage disease. J Geriatr Psychiatry Neurol 1993;6:29-33.

29. Lyketsos CG, Steele C, Galik E et al. Physical aggression in dementia patients and its relationship to depression. Am J Psychiatry 1999;156:66-71.

30. Deutsch LH, Bylsma FW, Rovner BW et al. Psychosis and physical aggression in Alzheimer's disease. Am J Psychiatry 1991;148:1159-1163.

31. McShane R, Keene J, Fairburn C et al. Psychiatric symptoms in patients with dementia predict the later development of behavioral abnormalities. Psychol Med 1998;28:1119-1127.

32. Chemerinski E, Petracca G, Teson A et al. Prevalence and correlates of aggressive behavior in Alzheimer's disease. J Neuropsychiatry Clin Neurosci 1998;10:421-425.

33. Aarsland D, Cummings JL, Yenner G et al. Relationship of aggressive behavior to other neuropsychiatric symptoms in patients with Alzheimer's disease. Am J Psychiatry 1996;153:243-247.

34. Gormley N, Rizwan MR, Lovestone S. Clinical Predictors of aggressive behavior in Alzheimer's disease. Int J Geriatr Psychiatry 1998;13:109-115.

35. Porsteinsson AP, Tariot PN, Erb R et al. Placebo-controlled study of divalproex sodium for agitation in dementia. Am J Geriatr Psychiatry 2001;9:5866.

36. Street JS, Clark WS, Gannon KS et al. Olanzapine treatment of psychotic and behavioral symptoms in patients with Alzheimer disease in nursing care facilities: A double-blind, randomized, placebo-controlled trial. The HGEU Study Group. Arch Gen Psychiatry 2000;57:968-976.

37. Katz IR, Jeste DV, Mintzer JE et al. Comparison of risperidone and placebo for psychosis and behavioral disturbances associated with dementia: A randomized, double-blind trial. Risperidone Study Group. J Clin Psychiatry 1999;60:107-115.

38. De D, Rabheru K, Rasmussen A et al. A randomized trial of risperidone, placebo, and haloperidol for behavioral symptoms of dementia. Neurology 1999;53:946-955.

39. Ballard CG, Margallo-Lana M, Fossey J et al. A 1-year follow-up study of behavioral and psychological symptoms in dementia among people in care environments. J Clin Psychiatry 2001;62:631-636.
40. McKhann G, Drachman D, Folstein M et al. Clinical diagnosis of Alzheimer's disease. Report of the NINCDS-ADRDA Working Group. Neurology 1984;34:939-944.

41. American Psychiatric Association. Diagnostic and Statistical Manual of Mental Disorders, Revised, 3rd Ed. Washington, DC: American Psychiatric Press Inc, 1987.

42. Stern Y, Folstein M, Albert M et al. Multicenter study of predictors of disease course in Alzheimer disease (the 'Predictors Study'). I. Study design, cohort description, and intersite comparisons. Alzheimer Dis Assoc Disord 1994;7:3-21.

43. Richards M, Folstein M, Albert M et al. Multi-center study of predictors of disease course in Alzheimer's disease (the 'Predictors Study'): II Baseline findings. Alzheimer's Dis Rel Disord 1993;7:22-32.

44. Stern Y, Sano M, Paulson J et al. Modified mini-mental state examination. Validity Reliability Neurol 1987;37 (Suppl. 1):179.

45. Stern Y, Albert SM, Sano M et al. Assessing patient dependence in Alzheimer's disease. J Gerontol 1994;49:M216-M222.

46. Blessed G, Tomlinson BE, Roth M. The association between quantitative measures of senile change in the cerebral grey matter of elderly subjects. $\mathrm{Br} \mathrm{J}$ Psychol 1968;114:797-811.

47. Devanand DP, Miller L, Richards M et al. The Columbia University Scale for Psychopathology in Alzheimer's disease. Arch Neurol 1992;49:371-376.

48. Folstein MF, Folstein SE, McHugh PR. 'Mini-mental state'. A practical method for grading the cognitive state of patients for the clinician. J Psychiatr Res 1975;12:189-198.

49. Wechsler D. Wechsler Adult Intelligence Scale-Revised. New York: The Psychological Corporation, 1981.

50. Goodglass H, Kaplan E. The Assessment of Aphasia and Related Disorders, 2nd Ed. Philadelphia, PA: Lea \& Febiger, 1983.

51. Eastwood MR, Lautenschlaeger E, Corbin S. A comparison of clinical methods for assessing dementia. J Am Geriatr Soc 1983;31:342-347.

52. Hope T, Keene J, Fairburn CG et al. Natural history of behavioral changes and psychiatric symptoms in Alzheimer's disease. Br J Psychiatry 1999;174: 39-44.

53. Kraemer HC, Yesavage JA, Taylor A. Can we learn about developmental processes from cross-sectional studies, or can we? Am J Psychiatry 2000;157: $163-179$. 